\title{
Self-adaptive grids for noise mapping refinement
}

\author{
C. Asensio*, M. Recuero, M. Ruiz, M. Ausejo, I. Pavón \\ Universidad Politécnica de Madrid (CAEND), c/Serrano 144, 28006 Madrid, Spain
}

\begin{abstract}
A B S T R A C T
Noise maps are usually represented as contour or isolines maps describing the sound levels in a region. Using this kind of representation the user can easily find the noise level assigned to every location in the map.

But the acoustic calculations behind the map are not performed for every single location on it; they are only performed in a grid of receivers. The results in this calculation grid are interpolated to draw the isolines or contours. Therefore, the resolution of the calculation grid and the way it was created (rectangular, triangulated, random...) have an effect on the resulting map.

In this paper we describe a smart iterative procedure to optimize the quality of the map at a really low additional computational cost, using self-adaptive grids for the acoustic calculations. These self-adaptive grids add new receivers to the sampling grid in those locations where they are expected to be more useful, so that the performance at the output of the interpolator is enhanced.

Self-adaptive sampling grids can be used for minimizing the overall error of the map (improving its quality), or for reducing calculation times, and can be also applied selectively to target areas or contour lines. This can be done by the user customizing the maximum number of iterations, the number of new receivers for each iteration, the target isolines, the target quality.
\end{abstract}

Keywords:

Noise mapping

Grid refinement

Uncertainty

Interpolation

GIS

\section{Introduction}

A noise map is a graphic representation of the sound level spatial distribution in a region, which is usually represented using contour lines. Nowadays, calculation tools based on noise models are widely extended for such maps, because of their high accuracy, the lower cost and the possibility they provide for the evaluation of different scenarios.

The acoustic calculations are performed in a grid of receivers distributed all over the location; this is a sampling calculation grid. Traditionally, this grid can be defined as a rectangular, triangulated or random grid [1].

It is necessary to calculate the sound level (Lk), usually Aweighted continuous sound pressure level (dBA), for every point $(k)$ in the grid. This is performed according to a specified acoustic model, usually with the help of some commercial software (Cadna, INM, Lima...). The uncertainty of the noise level calculations and models has been widely studied [2-16], and is beyond the scope of this paper.

A full map for the whole area is obtained by spatial interpolation of the results in the grid. There are several interpolation algorithms that can be used to draw the map: IDW, kriging. . [ $[1,17,18]$. The techniques for spatial interpolation can be divided into two main groups; deterministic and geostatistical interpolators. Deterministic interpolators create a surface from measurements based on the extent of similarity (inverse distance weighted) or the degree of smoothing (radial basis functions) of the data. Geostatistical interpolators (kriging) use least-squares regression algorithms to create a statistical model for the observed points, which allows the prediction of noise levels at unobserved (uncalculated) locations, leading to better results when applied to noise mapping.

Other acoustic considerations can be taken to improve the local performance of the interpolation process, for instance near barriers, or near sound sources [5].

After interpolation, it is necessary to make a classification in ranges (usually $5 \mathrm{~dB}$ ranges [19]), to get the isolines of the map. Fig. 1 shows the results $\left(L_{k}\right)$ at the receivers in the grid $(k)$, and the isolines obtained after interpolation and classification. Fig. 2 shows the full process of drawing up a noise map.

Obviously, the resolution of the calculation grid and the number of receivers in it have an influence on the concluding map. If we select a high-resolution grid, the isolines will be more accurate and precise.

Outdoor noise simulation software can perform all this process, making it all very easy for the end-user. The practitioner will have to select the size of the grid, as a compromise between the accuracy of the map, and the calculation effort.

In this context, it is a common practice when noise mapping large areas, to approach the calculation in two stages. The first 


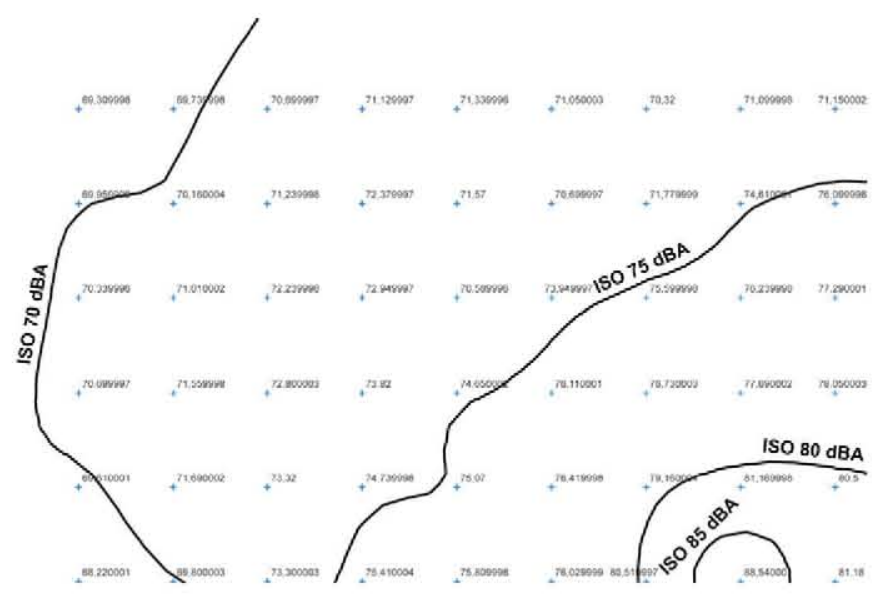

Fig. 1. Contour lines obtained by interpolation of sampling grid.

stage uses a coarse grid (for instance $30 \times 30 \mathrm{~m}$ ), to find the areas of interest. The second stage applies a higher resolution grid (usually $10 \times 10 \mathrm{~m}$ ), which is only applied to specific areas of interest, usually in populated areas. Occasionally thinner grids can be applied [19].

Following a similar strategy, the INM uses irregular sampling grids for noise mapping refinement [20].

The main objective of this paper is to present an efficient method that substantially improves the quality of a noise map (the precision and accuracy of its contour lines), by reducing the error derived from the calculation grid, and the interpolation + classification process, at a very low additional computational cost. This method estimates the uncertainty of the isolines in the map, and dynamically adapts the grid for the acoustic calculations, creating what we have denominated self-adaptive grids.

\section{Methodology}

The self-adaptive grids method is based on the following basis: The isolines are the supporting objects in a noise map, so that if the isolines are perfectly drawn, the map is completely perfect.

Assuming that this is true, we can derive that the error at any location between two consecutive isolines will be zero. So, the uncertainty in a noise map is produced by the random deviations between the noise level expressed by the isoline and the real value at each location on that line (Fig. 2).

Bearing this in mind, we can minimize the map's uncertainty by setting the focus on finding the correct location of the isolines, instead of getting information about the whole area. We must extend the grid of receivers to perform calculations at those locations where we can extract really useful information, avoiding useless receivers.

The idea behind this algorithm is to utilize uncertainty sampling for data exploitation [21], which is a widely extended concept in the field of active machine learning [22].

\subsection{Uncertainty in noise map isolines}

When we finally get a noise map, it will have several contour lines. If the accuracy and the precision of the map is optimal, these isolines would have been drawn at their correct position. The actual distribution of the error along the contour lines will cause uncertainty, as the true value for every position on the line is not known.

For instance, if the uncertainty of the "isoline $50 \mathrm{~dB}$ " is $\pm 1 \mathrm{~dB}$ for a level of confidence of $X \%$, it means that the true value along the $X \%$ of its length lies within the interval [ $49 \mathrm{~dB}, 51 \mathrm{~dB}$ ], therefore, the error on the $X \%$ of the length of the isoline will be lower (or equal to) than $1 \mathrm{~dB}$. Thus we can consider that the isolines are the supporting objects in the noise map, and we can use them to derive the quality of the map.

Following the GUM [23]) definitions, we can derive that for the calculation of the uncertainty it is necessary to establish a $95 \%$ confidence interval for the error on the isolines. The uncertainty of the concluding map will be affected by several factors like the quality of the input data [2], the acoustic model used for the calculations [11], the propagation of the uncertainty through the model [8]... On the other hand, the calculation grid and the interpolation process will also influence the uncertainty of the map, their being factors that the user can customize. Quantifying this contribution can be very important, as it can be minimized without changing the data inputs to the model. For instance, a very simple way to do it is by just using a thinner calculation grid, including more receivers. However, it would considerably increase the computational effort and the calculation times $[5,14]$.

In this paper we set the focus on the influence of the sampling grid, as the objective is to create an optimized self-adaptive grid that can improve the quality of the map at a low computational cost. All the other contributions to uncertainty of the calculations have been neglected, so we will consider that the results $L_{k}$, calculated by the acoustic model for the sampling grid, are true values.

\subsection{Measuring the quality of the map}

Although it is not strictly mandatory for running the self-adaptive grids algorithm, it was considered quite valuable to define an indicator that describes the quality of the map, as it can be used for taking decisions during the execution of the algorithm. This indicator will also be used for the assessment of the results, as a comparison of the quality of different maps.

There are several indicators that could have been selected: mean square error, differences of quantiles [24], but we preferred to use the uncertainty on the isolines as described in [25], and summarized in this section, because it more properly weights the bias and the variability along the isoline, taking into account the number of observations. Even in the event of the conditions for this parametric approach not being met, it gives a good measure of the quality of the map that can be used for comparison purposes, or to control the flow of the algorithm.

After having drawn up the map, we will have several contour lines in $5 \mathrm{~dB}$ ranges (usually). We will consider a single contour

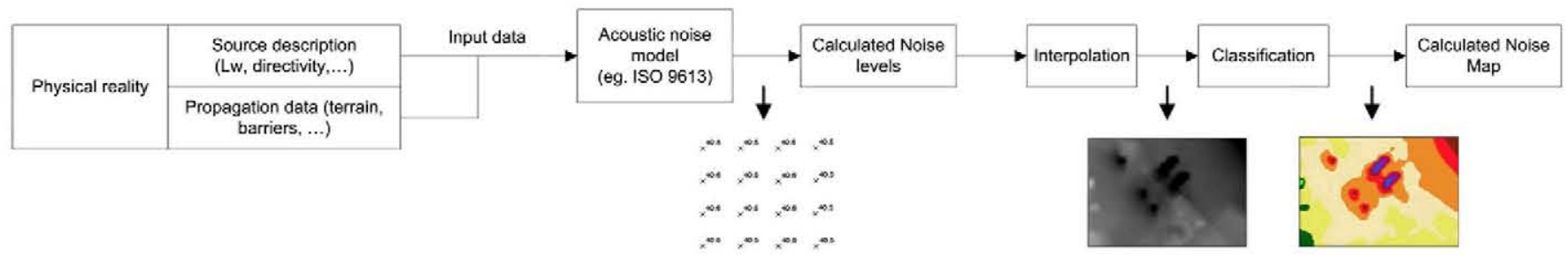

Fig. 2. Process of drawing up a noise map. 


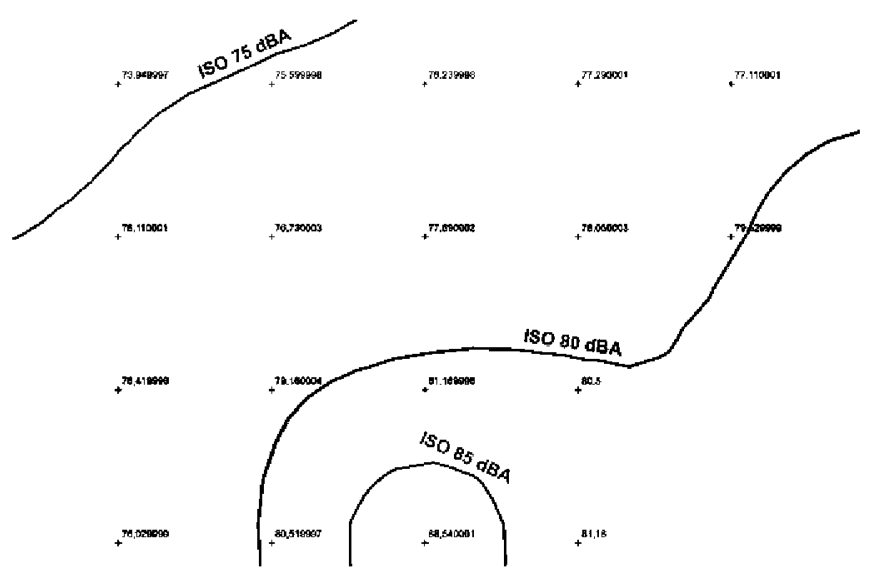

Fig. 3. Isoline of a noise map.

line in this map (see Fig. 3). Every location in the isoline has been assigned an interpolated value of $L_{\text {iso }} \mathrm{dB}$. But, if we used the acoustic model for the calculation of the true values at these locations (Li), we would find a difference with respect to $L_{i s o}$, which is the interpolation error at each point $\left(e_{i}\right)$.

$e_{i}=L_{\text {iso }}-L_{i}$

The random variable $E$ describes this error along the contour line, that is assumed to be normally distributed (central limit theorem) according to the following equation:

$E \rightarrow N(\mu, \sigma)$

The physical and spatial meaning of $\mu$ (Fig. 4) is related to the balance between the interpolated and the true isolines. If the calculation grid has been thin enough, this bias should be very small $(\mu=0)$, otherwise the bias could be corrected to improve the quality of the map.

On the other hand, the variance $\left(\sigma^{2}\right)$ is related to the variability of the error along the line, so it has a contribution to the uncer-

interpolated

$$
\begin{array}{r}
\mu \neq 0 \\
\sigma=0
\end{array}
$$
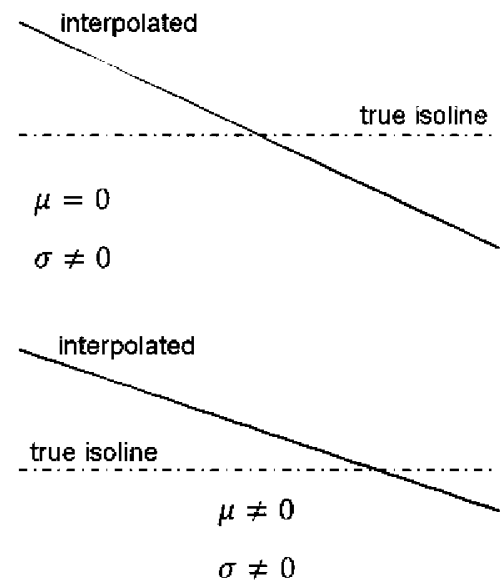

Fig. 4. Spatial effect of the $\mu$ and $\sigma^{2}$ tainty. The variance is related to the anti-parallelism between the true and the interpolated contour lines (Fig. 4).

$95 \%$ of the length of the isoline will have an error within the interval:

$[\mu-1.96 \sigma, \mu+1.96 \sigma]$

As it is not a feasible option to change the value of the isolines (i.e. $59.56 \mathrm{~dB}$ ), we cannot make a correction for the bias, so it must be included as a contribution to uncertainty:

$U= \pm[|\mu|+1.96 \sigma]$

The parameters $\mu, y, \sigma$ are not known, consequently, it is necessary to make an estimation from data observations. For this purpose, we must create a set of $\mathrm{n}$ sampling points on the contour line, and make noise calculations using the acoustic model $\left(L_{i}\right)$ at those specific locations (Fig. 5).

Using the new true values, we will get error observations; we will estimate the mean and the variance using Eqs. (5) and (6), respectively.

$\bar{e}=\frac{\Sigma_{i} e_{i}}{n} \quad i \in 1 \ldots n$

$\hat{s}^{2}=\frac{1}{n-1} \sum_{i=1}^{n}\left(e_{i}-\bar{e}\right)^{2} \quad i \in 1 \ldots n$

Each time we estimate the parameters from observations, we would obtain different estimations, because of the randomness. Because of this, it is important to get a confidence interval for the parameters.

The bilateral $95 \%$ confidence interval for $\mu$ is:

$\mu \in \bar{e} \pm t_{n-1,2.5 \%} \cdot \frac{\hat{s}}{\sqrt{n}}$

and the unilateral $95 \%$ confidence interval for $\sigma^{2}$ is:

$\sigma^{2}<\frac{(n-1)}{\chi_{n-1,5 \%}^{2}} \cdot \hat{s}^{2}=\hat{s}_{\text {max }}^{2}$

We can estimate the expanded uncertainty using:

$U= \pm\left[|\bar{e}|+\hat{s} \cdot \frac{t_{n-1,2,5 \%}}{\sqrt{n}}+1.96 \hat{s} \cdot \sqrt{\frac{(n-1)}{\chi_{n-1,5 \%}^{2}}}\right]$

From Eq. (9), we can derive that the uncertainty of the analyzed isoline depends on the mean error, on the variability of the observations and on the number of measurements. As stated previously, this indicator $U$ is more suitable for the creation and evaluation of self-adaptive grids, as it considers and properly weights the number
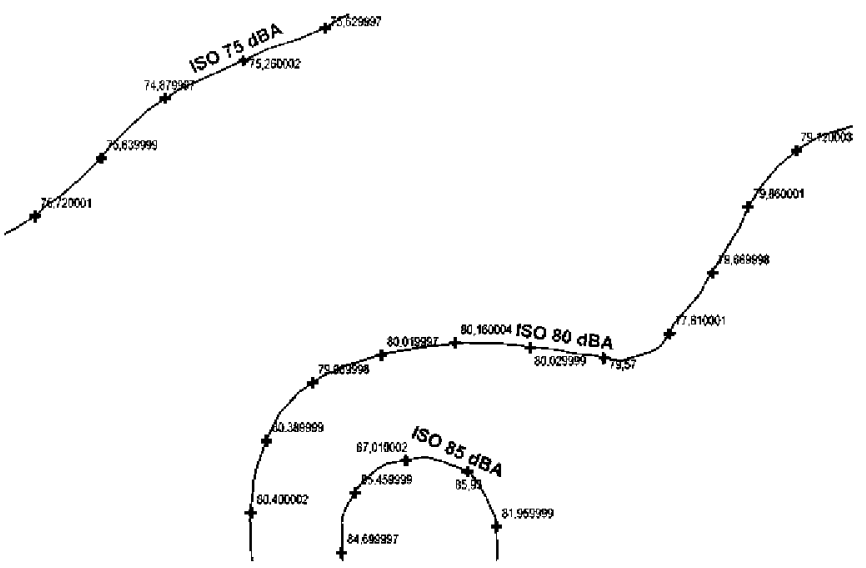

Fig. 5. Receivers used for the estimation of uncertainty. 
$50-55 \mathrm{~dB}$

- $55-60 \mathrm{~dB}$

True isoline

— Interpolated isoline

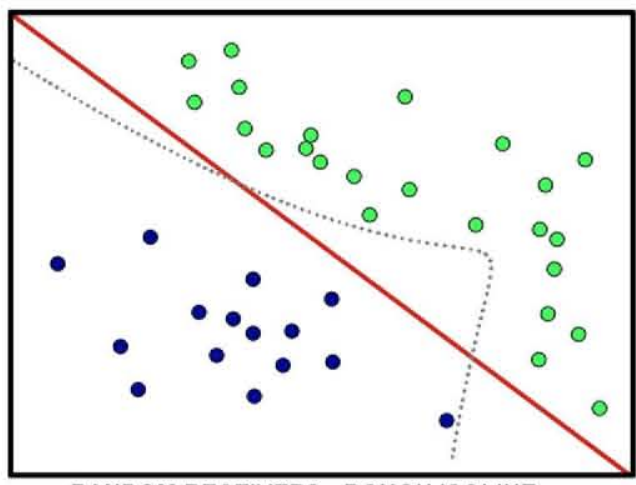

RANDOM RECEIVERS - ROUGH ISOLINE

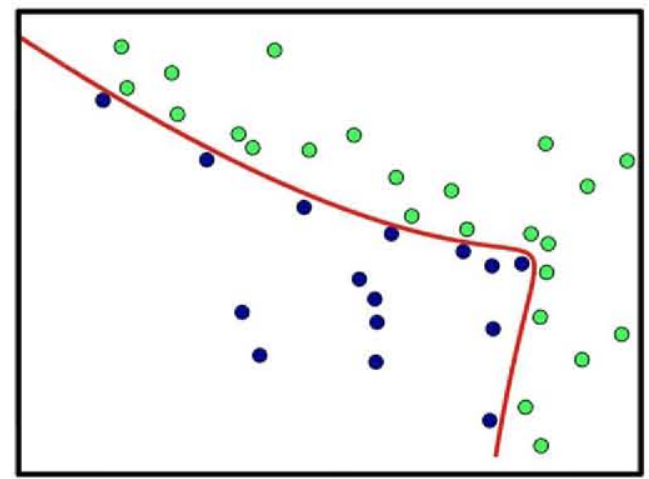

SMART RECEIVERS - REFINED ISOLINE

Fig. 6. The effect of the sampling grid for the interpolation results.

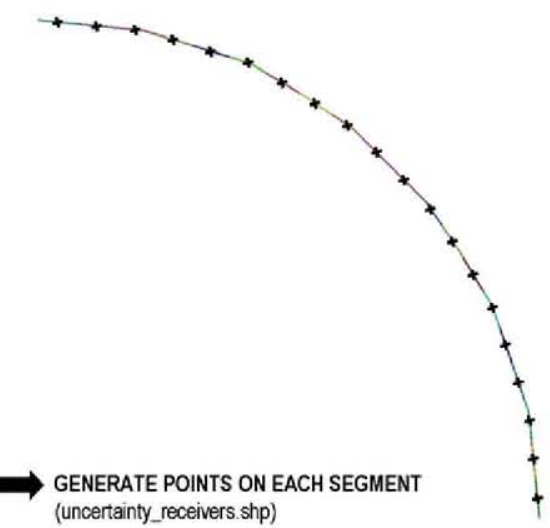

Fig. 7. Creation of uncertainty receivers.
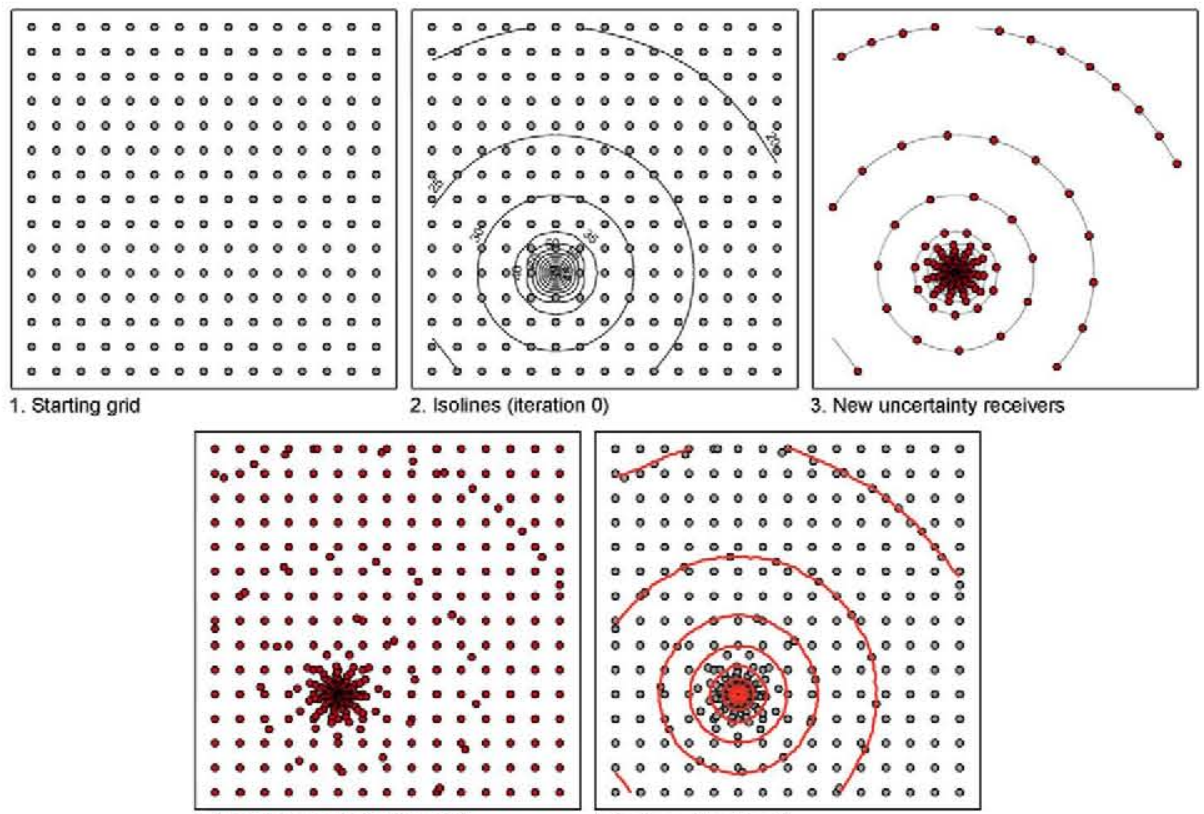

4. Iteration 1 self-adaptive grid

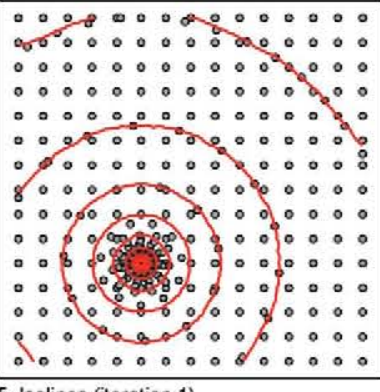

5. Isolines (iteration 1)

Fig. 8. Construction of self-adaptive grids. 


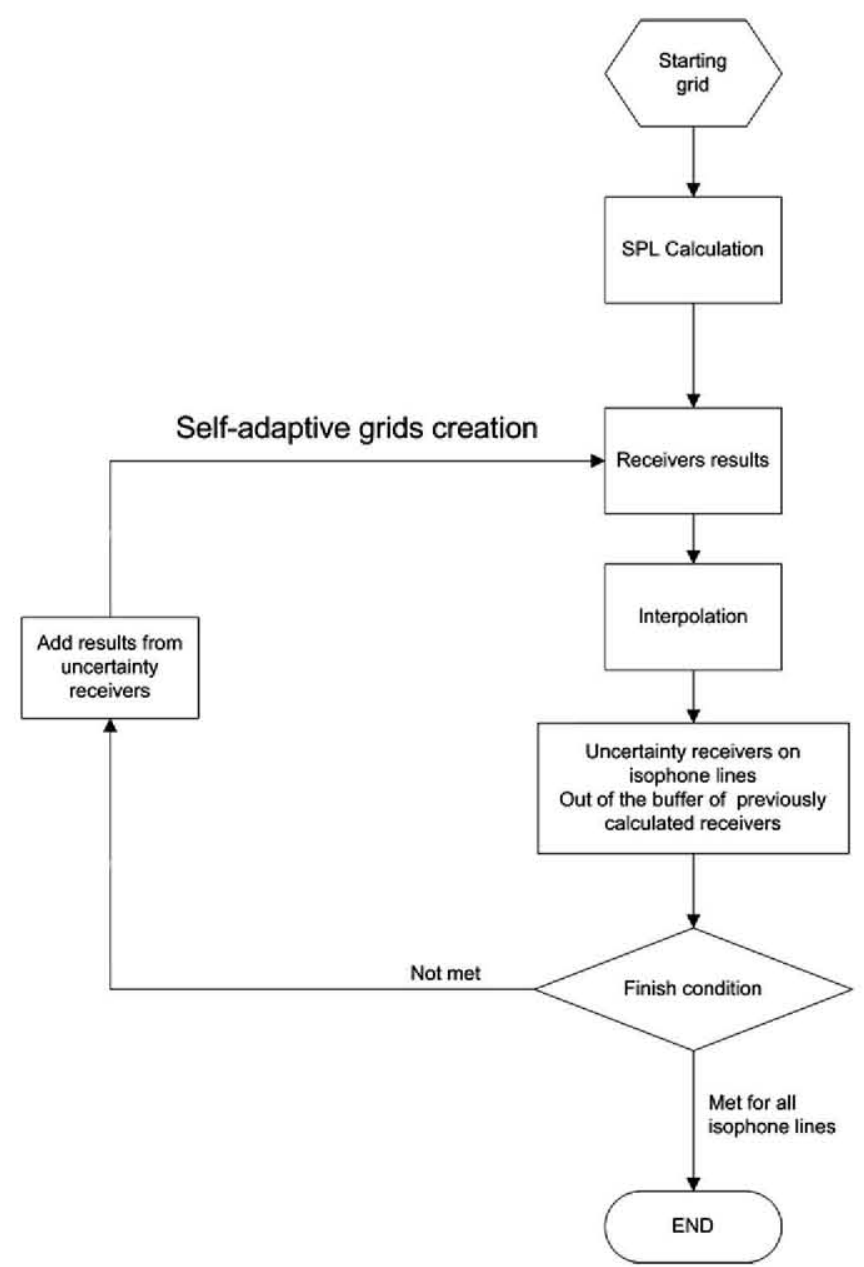

Fig. 9. Procedure for the creation of self-adaptive grids. of observations in the isoline ( $n$ ) affecting the mean error and the variability of error.

\subsection{Noise mapping using self-adaptive grids}

To start the practice it is necessary to make a first calculation according to the classical procedure (this is iteration 0 ). The starting grid will be a compromise between accuracy and computational cost, and it can be rectangular, triangulated or random. After calculation (using the noise model), interpolation and classification (using GIS), we got a noise map of a quality that we wished to improve.

To improve the quality of the map, it is necessary to improve the quality of the contour lines; subsequently it is necessary to reduce their uncertainty $(U)$. In order to do so, we must add extra information to the calculation grid at the input of the interpolator; consequently, it is necessary to perform new acoustic calculations for new locations.

The key to this algorithm is to properly select the locations for these new receivers. The location of the new receivers is really important; as some locations can add more useful information to the map (Fig. 6 illustrates the performance of the interpolator for two different sets of input data). We will try to select those locations that are expected to be more useful, which is along the contour lines. In consequence, by trying to exploit the data inputs to the interpolator, we will add new receivers "on" the isolines (Fig. 5), and we will make them useful in two different ways:

- They can be used to make an estimation of the quality of each isoline in the previous iteration (uncertainty receivers)

- They will be added to the starting grid for a new spatial interpolation.

The new uncertainty receivers are created using simple GIS tools (for instance using Esri Arcmap ([18]) the line can be divided using regular segments into a point shape file, either by

Table 1

Number of receivers involved in the scenario "point sound source in an open space".

\begin{tabular}{|c|c|c|c|c|c|}
\hline & $10 \times 10 \mathrm{~m}$ Rectangular grid & Iteration $0100 \times 100 \mathrm{~m}$ rectangular starting grid & Iteration 1 & Iteration 2 & Iteration 3 \\
\hline Receivers calculated in the acoustic model & 196348 & 1962 & 306 & 47 & 42 \\
\hline Receivers in the interpolation grid & 196348 & 1962 & 2268 & 2315 & 2357 \\
\hline
\end{tabular}

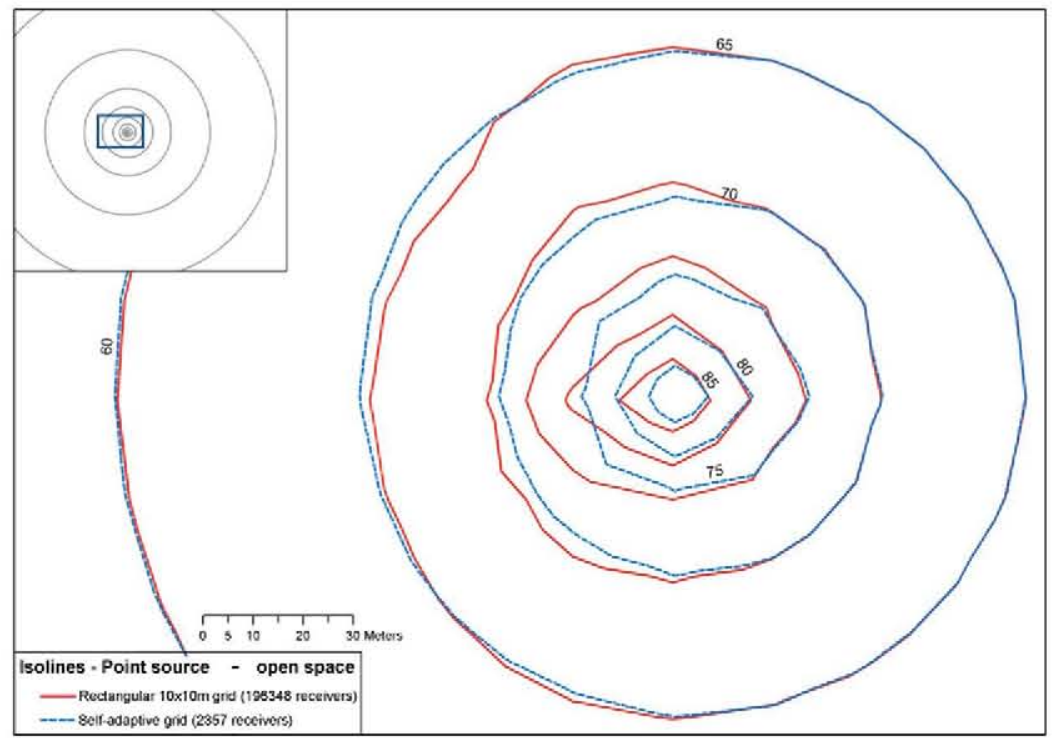

Fig. 10. Self-adaptive grids performance for a point source scenario. 
a number of divisions or distance, as shown in Fig. 7). The user should decide the number of receivers or the distance between them for every iteration, bearing in mind that in this iterative process the lack of information can be compensated in the following iterations.

Then, the quality of the map might be evaluated according to the procedure described in the previous sections. Afterwards, the self-adaptive grid starts self-creating.

Adding the new uncertainty receivers to the starting grid, we can interpolate a new map (iteration 1). So, the grid grows iteratively, adding the new uncertainty receivers that are located on the isolines from the previous iteration.

So for every iteration, the quality of the isolines in the previous iteration are estimated, and a new enhanced map is created taking advantage of the receivers used for the quality assessment.

Every new map has been created using all the receivers in the previous grid, and extra receivers, so it will be more accurate than the previous one, as the interpolator has made its prediction from an enhanced data input (self-adaptive grid). Fig. 8 illustrates the process of creation of the new self-adaptive grid, and Fig. 9 shows the flow chart.
Note that the goal of this procedure is to concentrate the computational effort near the noise isolines, as the information gathered in this region is more helpful.

In this way the starting grid can be selected thicker than what is required according to classical procedures, as the following iterations will improve the quality of the map.

Although it can be useful to estimate the uncertainty as a method to quantify when the map is good enough, the estimation of the uncertainty is not strictly a goal in this algorithm. For this reason, it is possible to simplify the process by just skipping calculations in buffer areas around previously calculated receivers. This is a way to prevent calculating redundant receivers, although it might alter the estimation of the uncertainty. A small radius should be selected (for instance $2 \mathrm{~m}$ ) for those scenarios where there are narrow streets, but it can be larger in countryside scenarios (up to $15-20 \mathrm{~m}$ ).

Self-adaptive grids can be very effective in the first iterations. As the isolines become more accurate, the new receivers provide less new information, so, it must be taken into account in order to define the conditions that stop the iterative algorithm.

\section{Point source - open space}

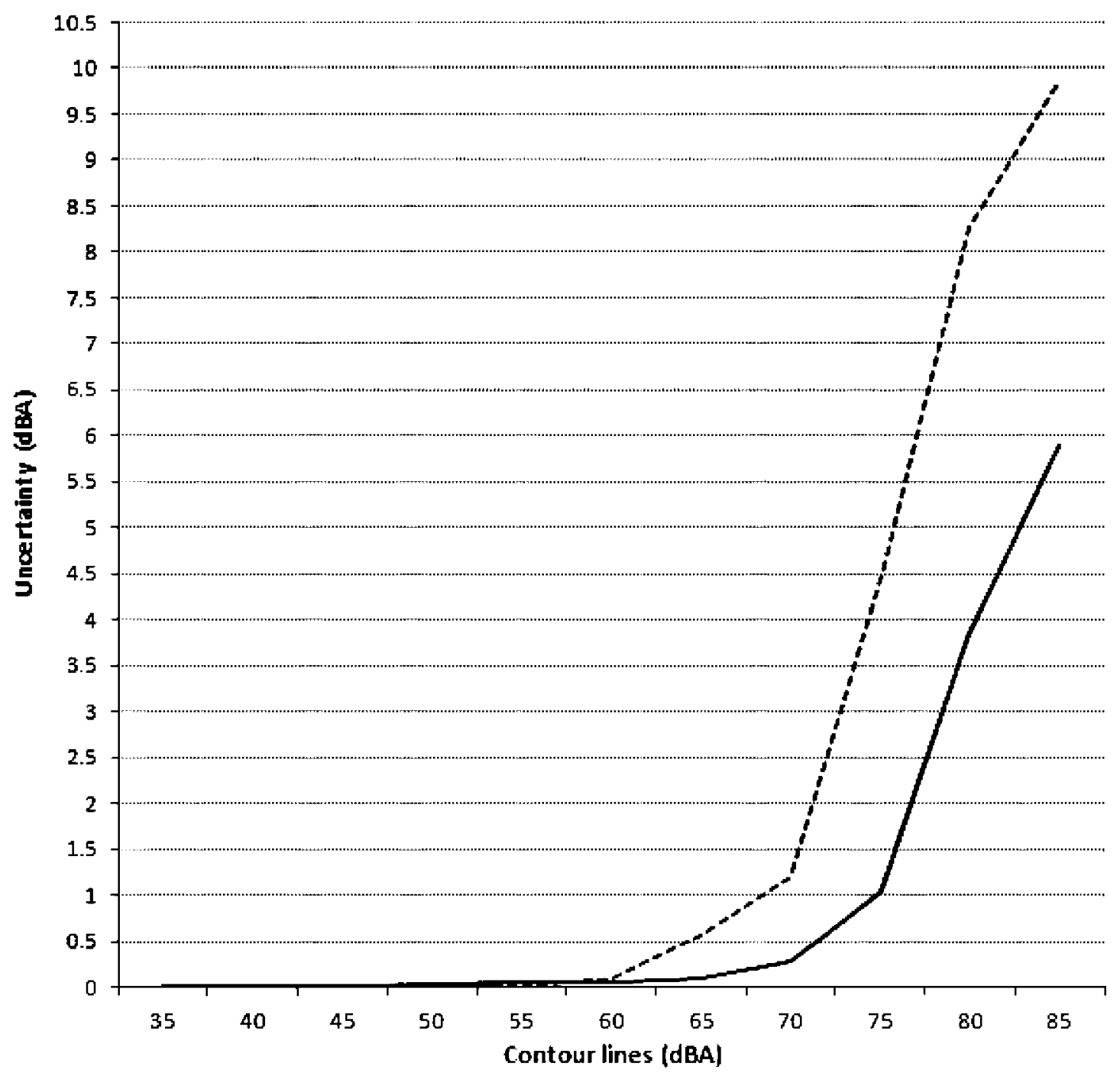

Fig. 11. Uncertainty enhancement achieved by self-adaptive grids in a point source scenario. 
Some of the possibilities for finishing conditions are the following:

- The uncertainty is lower than a user-defined threshold. For instance, if the threshold is independent for each isoline, the user will be able to set the focus on those isolines of interest for action planning.

- The maximum number of iterations has been reached. This option limits time consumption.

- The improvement in the quality of the line is negligible. This will happen when the noise isolines are already well fitted.

- Most of the length of the noise isoline has been previously calculated. If we want to prevent the creation of redundant receivers (using a buffer around previously calculated receivers), perhaps there is no room for new receivers.

- The calculated isoline is very near its true location, closer than a user-defined threshold. When we observe an error $(\mathrm{dB})$ at a certain location on the isoline, it means that the isoline was not correctly drawn, so we could estimate how deviated the interpolated contour lines are from the true one. Using a GIS tool we can derive the slope of the $\operatorname{map}(\mathrm{dB} / \mathrm{m})$ at each point, and use it to transform the error in terms of level differences into maximum error in terms of distance. In this way we could estimate the quality of the map as a confidence interval for the distance between the true and the interpolated isoline.

- Other criteria can be used as a function of the expected output results and the target quality of the map [26].

\section{Results}

The benefits obtained by using self-adaptive grids depend on each case, as many factors are involved: the number of sources, the source's strength ratio at each receiver, source-receiver distance, the presence of obstacles, the starting grid (which is often a project requirement), or even the size of the simulation. Even the calculation times for each receiver can be very different depending on the software used, the model applied, the parameters for the calculation engine, the characteristics of the computer used...

To illustrate the performance of the self-adaptive grids, we show results for some representative examples: a simple point source scenario, a complex multi-source industrial site and a case in which only one contour line is of interest. Acoustic models and calculations have been carried out with Lima 5.02 ([27]), a software package used primarily for outdoor noise prediction. However, this tool does not allow the interpolation of irregular grids, so ESRI ArcInfo Spatial Analyst was used for spatial interpolations.

All the calculations were performed in a PC running Windows $\mathrm{XP}$ (dual Xenon processors $3 \mathrm{GHz}, 3 \mathrm{~GB}$ RAM), using the default calculation options in Lima for the ISO 9613 noise model. As the process includes commercial software packages, it was not possible to make a full automatic process, so the reference time intervals in this paper refer to the calculation times in Lima (acoustic model calculations).

\subsection{Point sound source in an open space}

The first example shows the results obtained on the simpler scenario: a single point sound source in an open space.

We used a $100 \mathrm{~m}$ equally spaced starting grid to obtain the first noise map (iteration 0 contains 1962 receivers). In order to calculate the uncertainty of this map, a new set of simulation receivers was located on every isoline, and the calculation was repeated. Using this new set of results, the uncertainty of each line was calculated using Eq. (9). The number of equally spaced new receivers at each isoline was decided according to the following expression:

$$
\max \left(2 \cdot \frac{\text { isophone line length }}{\text { grid size }}, 10\right)
$$

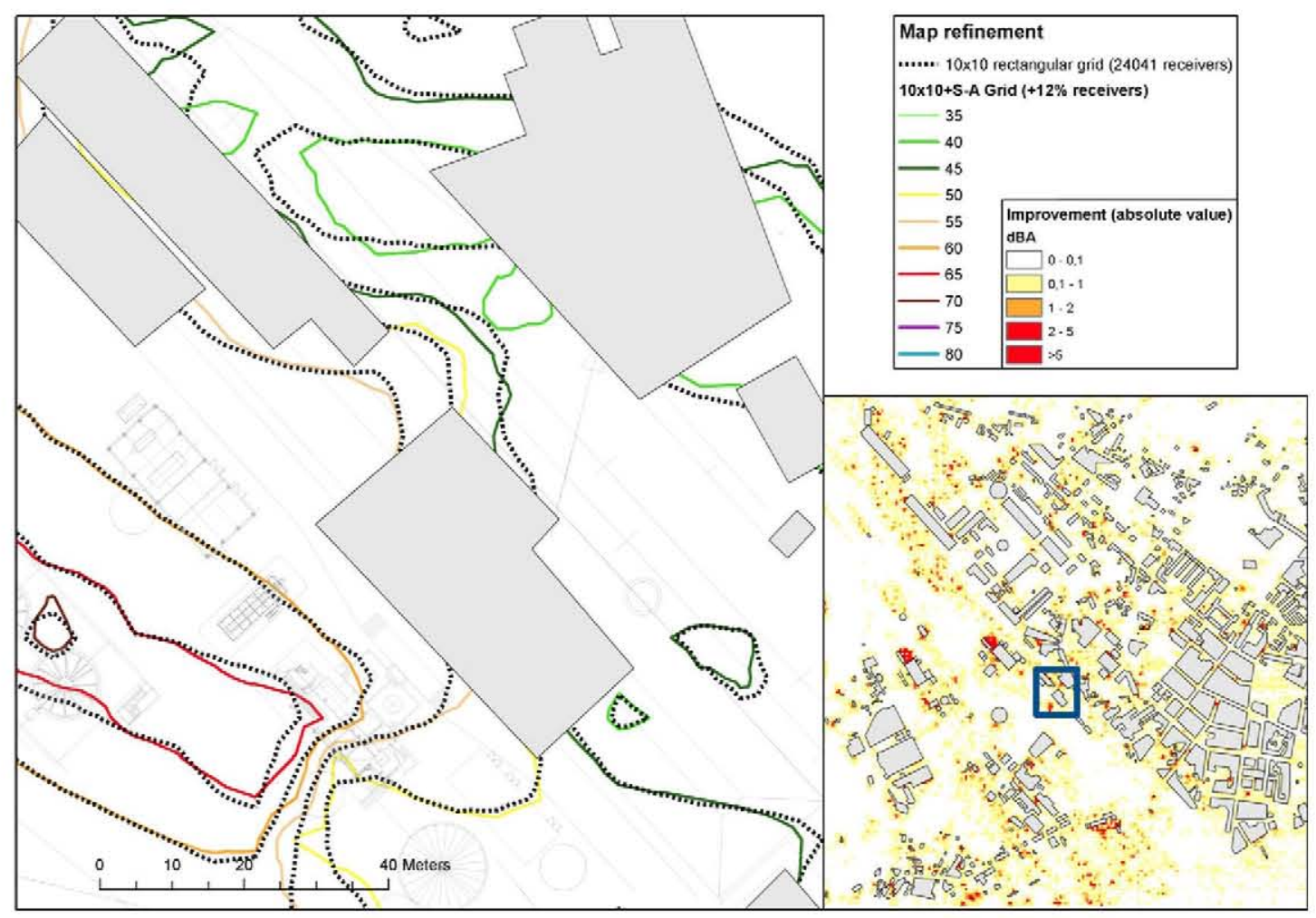

Fig. 12. The effect of self-adaptive grids in a multi-source industrial site. 
In this example, it must be noted, that when the uncertainty of a given isoline is low enough it is excluded for the next iteration.

Table 1 shows, for each iteration and for a reference $10 \times 10$ rectangular grid, the number of receivers calculated, and the number of receivers in the grid.

Fig. 10 zooms the area near the source to show the differences in the contour lines for three different grids: $1 \times 1 \mathrm{~m}$ rectangular grid, $10 \times 10 \mathrm{~m}$ rectangular grid, and a self-adaptive grid with three iterations, and Fig. 11 compares the uncertainty of the isolines.

Self-adaptive grids have shown a considerably better performance at the contour lines over $60 \mathrm{~dB}$, while their performance is almost the same when obtained using a high-resolution grid for the further isoline. The quality of the map is better with a self-adaptive grid and it was created using only $1.2 \%$ of receivers.

Time efficiency cannot be seriously evaluated as this is such an easy case that the acoustic software took approximately $20 \mathrm{~s}$ to calculate the 200,000 receivers in the rectangular $10 \times 10 \mathrm{~m}$ grid.

\subsection{Multi-source industrial noise}

In the case of multisource scenarios or in the presence of many obstacles, the starting grid might be of a higher resolution so that

Industrial multi-source site

- Self-adaptive grid (12143 receivers)

$10 \times 10$ rectangular grid (24041 receivers)

---.- Self-adaptive grid (29684 receivers)

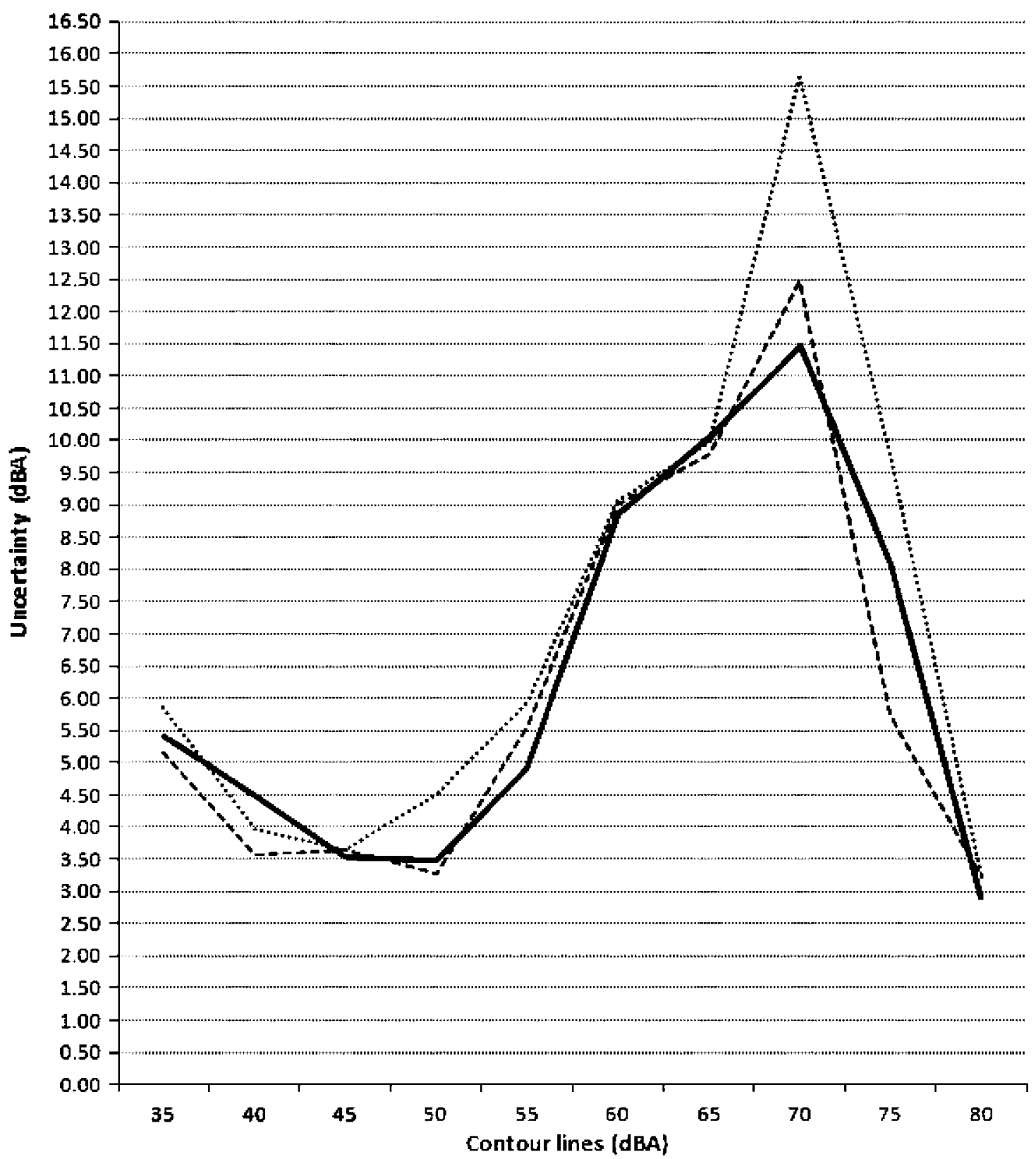

Fig. 13. Uncertainty enhancement achieved by self-adaptive grids in a multi-source industrial site. 
gaps can be detected. In this particular case, it was found that a 5$10 \%$ increase in the number of receivers used can reduce the uncertainty substantially in the starting grid.

In the following example, a $10 \mathrm{~m} \times 10 \mathrm{~m}$ initial grid has been chosen to make a noise map. After just one iteration, the addition of $12 \%$ receivers (from 24,041 to 26,829 ), improves results in those areas of the map closer to buildings and sound sources (colored area in Fig. 12, right). This improvement is only significant in relation to the refinement gained at the location of the isolines (Fig. 12, left) and the enhancement produced in their standard uncertainty (Fig. 13).The uncertainty of the lines placed closer to the noise source has been improved notably (up to $5 \mathrm{~dB}$ ),

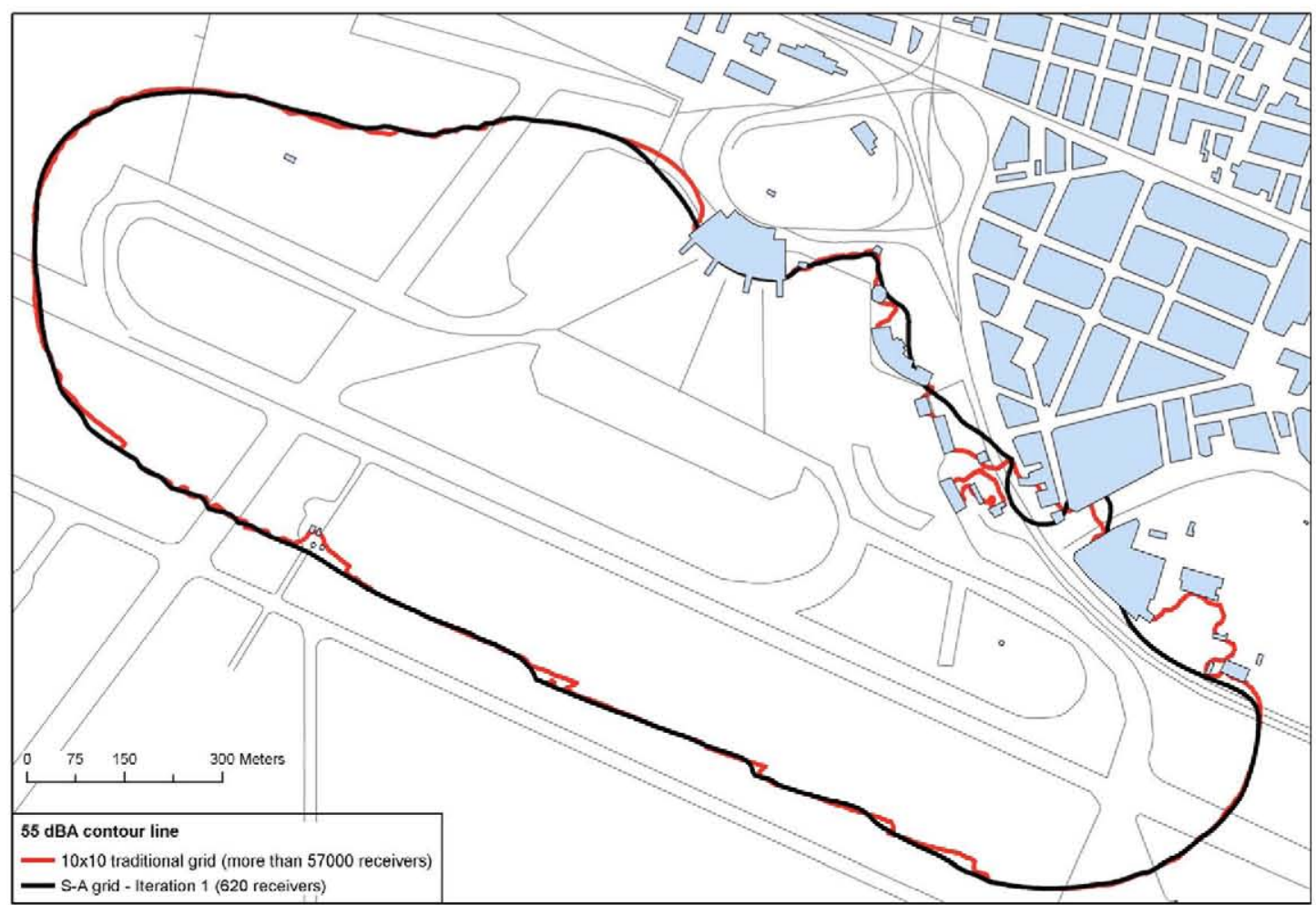

Fig. 14. Problems detected using regular self-adaptive grids for refinement of a single contour line.

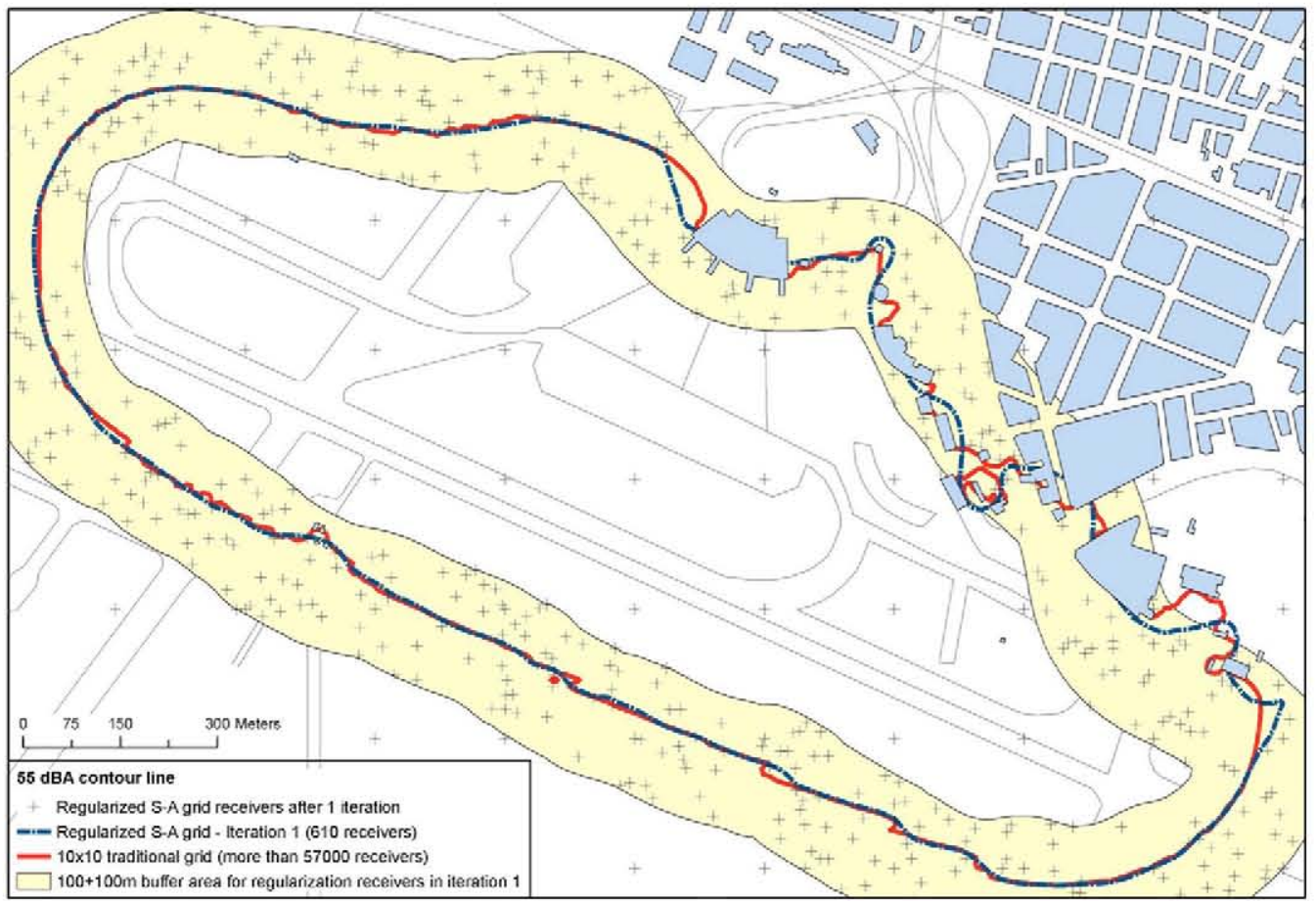

Fig. 15. Regularization in self-adaptive grids. 


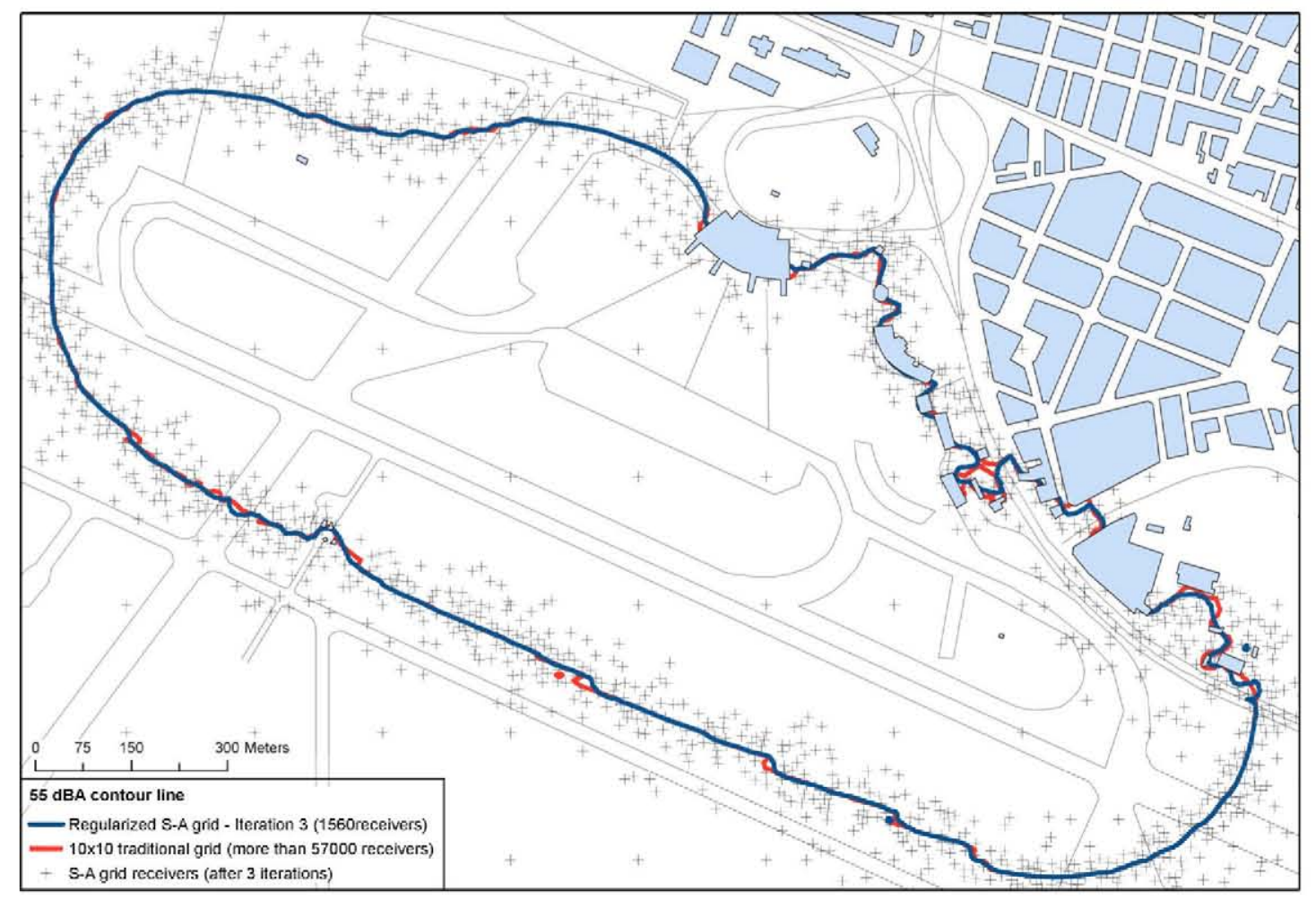

Fig. 16. Regularized self-adaptive grid after 3 iterations.

unlike farther away lines, which see an improvement of less than $0.5 \mathrm{~dB}$.

We tried the same scenario using a $25 \times 25$ starting grid. After three iterations, approximately 12,000 receivers had been calculated. Although we used a half of receivers, the results are better than those calculated from the rectangular grid for almost every contour line (Fig. 13).

\subsection{Special cases}

A different approach must be considered when only one contour line is of interest. In the following example, the focus is set on the $55 \mathrm{dBA}$ isolines for the night period, which is the line that establishes the area where buildings must be considered for action planning in relation to taxiway noise in an airport. The accuracy and the precision of this contour line must be high, but the rest of the map is not so important, therefore we applied a regularized version of the self-adaptive grid algorithm.

In this example the starting grid was a $200 \mathrm{~m}$ rectangular grid, containing 145 receivers. After interpolation, we only drew the $55 \mathrm{dBA}$ contour line. This was an approximately $5 \mathrm{~km}$ length isoline that we decided to sample using new receivers separated $10 \mathrm{~m}$. The first iteration added 475 receivers, which achieved quite a good performance (for a total of 620 receivers) compared with those obtained from a $10 \times 10$ rectangular grid, which contains more than 57,000 receivers. But we noticed that the performance is not as high in those areas closer to buildings (Fig. 14). The following iterations do not solve the problem efficiently, because the new information becomes redundant as all the receivers are created close to the only isoline considered.

In order to solve this problem, the first iteration in the creation of self-adaptive grids must implement a regularization process, adding random receivers in a buffer area around the contour line. So, we created a $200 \mathrm{~m}$ buffer around the isoline (from iteration 0 ), to add 475 new regularized random receivers that were used to interpolate the new contour line (Fig. 15). This simple process
Table 2

Performance of regularized self-adaptive grids.

\begin{tabular}{lll}
\hline & Regularized S-A grid & Rectangular 10 × 10 grid \\
\hline Iterations & $1+3$ & 1 \\
Number of receivers & 1560 & 57000 \\
Calculation time (min) & 1 & 15 \\
Maximum error (dBA) & 6.650 & 8.87 \\
Minimum error (dBA) & -4.940 & -2.24 \\
Mean error (dBA) & 0.012 & -0.042 \\
Standard deviation (dBA) & 0.867 & 0.740 \\
Uncertainty (dBA) & 1.885 & 1.635 \\
\hline
\end{tabular}

allows a better performance of the following iterations in those areas near buildings. The second iteration added 475 on the isoline obtained in iteration 1 (only iteration 1 added random regularized receivers). The third iteration added 475 new receivers on the contour line from iteration 2. For a total number of 1560 receivers, we achieved almost the same $55 \mathrm{dBA}$ contour line as using a $10 \times 10$ rectangular grid containing more than 57,000 receivers (Fig. 16). As shown in Table 2, the uncertainty of the map is almost the same, and the maximum and minimum error over the contour line is lower in the one calculated using self-adaptive grids. The 1560 receivers were calculated in approximately one minute (quite an easy case, using acceleration options in Lima), while it took more than $15 \mathrm{~min}$ to calculate the 57,000 receivers in the high-resolution grid (for the same setup in Lima).

The inconvenience of this regularization process is that it does not allow evaluating the uncertainty for the first iteration, as the receivers are not located on the contour line.

\section{Conclusions}

We have described a new iterative procedure used to optimize noise maps. This procedure takes advantage of the receivers involved in the estimation of the uncertainty of the isolines in the map, creating self-adaptive grids. 
After using a noise model to make acoustic calculations in the positions defined by the self-adaptive grid, spatial interpolation is performed to obtain enhanced map contour lines.

The main advantages of self-adaptive grids include the following:

- It is a smart procedure which analyses previous knowledge to concentrate the calculation effort in those areas where it will be more useful. Because of this, it can provide better results than higher resolution grids, while using fewer receivers for interpolation.

- Self-adaptive grids improve the results obtained by any starting grid, no matter what the resolution, the number of receivers, or the way they have been located (random, triangulated or equal spaced). Whatever the interpolation method used (inverse distance weighting, spline, kriging,..), their parameters or other constraints are also irrelevant, as all of these will improve the accuracy of results as further knowledge (extra receivers) is provided as inputs.

- Self-adaptive grids have been proven to be an excellent method for open spaces with few obstacles. The use of coarse initial grids, allow the number of calculated receivers to be reduced enormously, while improving the accuracy of the map. The calculation time is drastically reduced as the number of calculation receivers is reduced.

- This method may be very useful for grid refinement in scenarios where few obstacles (especially buildings and barriers) are considered. For instance, this is the case when noise mapping airports using INM [20].

- Even if a fine initial grid has been chosen, a single iteration of the method will improve the map's accuracy at a low computational cost. As a result, self-adaptive grids have also shown good results when applied to multi-source acoustic sites.

- The first iteration always allows a proper estimation of interpolation bias and uncertainty for the starting grid.

- Self-adaptive grids can be applied to specific isolines, allowing the quality of relevant isolines to be improved, for instance those used to define airport footprints.

The weak points of self-adaptive grids can be summarized as follows:

- Representation ranges must be chosen from the very beginning because the optimization process focuses on the chosen isolines. If new ranges are selected for visualization, the results will not be optimized, and the uncertainty calculation will be incorrect. This issue could be solved by using small ranges for the iterative process, and target ranges for the representation of the map, but obviously this process seems to be less effective.

- When there are many obstacles, the use of coarse starting grids may result in the gaps being missed.

- To reduce calculation times, it has been proposed to avoid creating new receivers close to those previously calculated. Therefore, uncertainty may not be estimated properly, as these buffers are excluded from calculations.

- When noise maps are calculated for more than one period (for instance, day, evening and night), self-adaptive grids should be used independently for each of these periods. This will improve the effectiveness of each iteration, but will increase the number of extra receivers.

\section{Further Work}

This paper can be considered as a proof of a concept. Further work is needed create a fully automated procedure that can be implemented in a noise mapping software.
Apart from this, some other issues are described in this section.

The finishing condition and buffer radius, mentioned in the algorithm, should be further studied in relation to the posterior use of commercial simulation software and according to the enduser's feedback. Depending upon the prediction scenario and map requirements, a selection of parameters that usually works can produce inappropriate results.

Starting grids are inevitably joined to self-adaptive grids, setting their effectiveness and usefulness. The coarser the starting grid, the better the improvement achieved by the self-adaptive grid, but the greater the possibility of missing gaps. For a finer initial grid, the self-adaptive grids will have little improvement in the results.

The ranges used during the creation of the self-adaptive grids have an influence on the behavior of the algorithm. Using $5 \mathrm{~dB}$ ranges requires fewer receivers at each iterative loop. However, using $1 \mathrm{~dB}$ ranges will make each loop more effective.

Self-adaptive grids can be slightly modified or combined with other techniques in order to achieve a better performance. For instance, in the case of setting the focus on just one contour line (airport footprint) a regularization process can be applied.

To achieve the best algorithm, the algorithm needs to be implemented in sound propagation calculation software and the results studied. The combined effects of the self-adaptive grids, ranges and other acoustic constraints need to be evaluated in order to find a good balance for the initial parameters.

The self-adaptive grids have been intended to work in noise maps accompanied by simulation tools. Moreover, self-adaptive grids can also be customized for measurement maps. However, the costs of making new measurements may make this unrealistic.

Other points of discussion and future research include the possibility of using self-adaptive grids for mapping, based on simulations or measurements, in other fields of science.

\section{References}

[1] de Smith MJ, Goodchild MF, Longley PA, Geospatial analysis. A comprehensive guide to principles, techniques and software tools. 3rd ed. SPLINT, London; 2009.

[2] Ausejo M, Recuero M, Asensio C, Pavôn I, Lôpez JM. Study of precision, deviations and uncertainty in the design of the strategic noise map of the macrocenter of the city of Buenos Aires, Argentina. Environ Model Assess 2010;15:125-35.

[3] Manvell D, Aflalo E. Uncertainties in environmental noise assessments - ISO 1996, effects of instrument class and residual sound, Forum Acusticum. Budapest, Hungary; 2005.

[4] De Muer T, Botteldooren D. Methods for quantifying the uncertainty in noise mapping. In: Symposium: managing uncertainty in noise measurement and prediction. Le Mans, France; 2005.

[5] Stapelfeldt H, Manvell D. Optimising uncertainty and calculation time. Forum Acusticum. Budapest Hungary; 2005.

[6] Payne R. Uncertainties associated with the use of a sound level meter, NPL. DQL-AC 002; 2004.

[7] Alberola Asensio J, Mendoza López J. Bullmore AJ, Flindell IH. Noise mapping: uncertainties. Forum Acusticum, Sevilla Spain; 2002.

[8] Shilton SJ, Van Leeuwen JJA, Nota R. Error propagation analysis of XPS 31-133 and CRTN to help develop a noise mapping data standard. Forum Acusticum, Budapest, Hungary; 2005.

[9] Shilton SI, Van Leeuwen IJA, Nota R. Accuracy implications of using the WGAEN good practice guide toolkits. Forum Acusticum, Budapest, Hungary; 2005

[10] Shilton S, van Leeuwen H. Research Project NANR 93: WG-AEN's good practice guide and the implications for acoustic. Final report: Quantified accuracy of GPG toolkits, Department for Environment, Food and Rural Affairs (DEFRA). DGMR V.2004.1300.00.R011.1; 2005.

[11] Shilton S, Trow J, Hii V, Archer N. Research Project NANR 208: Noise modelling. Final report - Part 5: quantified accuracy of GPG toolkits - CRN, The Department for Food and Rural Affairs (DEFRA). DGMR V.2006.1247.00.R4-5; 2007.

[12] De Muer $T$, Botteldooren D. Uncertainty in noise mapping: comparing a probabilistic and a fuzzy set approach, Lecture notes in computer science. In: Proceedings of the 10th international fuzzy systems association world congress conference on Fuzzy sets and systems, vol. 2715; 2003. p. 184.

[13] Probst W, Huber B. How to evaluate the accuracy of noise mapping calculations - the standardized approach according to DIN 45687 Internoise. Istanbul, Turkey; 2007.

[14] Stapelfeldt $H$, van Vanda $E H$, Manvell D. Quality assurance for the calculation of large noise maps. Forum Acusticum, Sevilla Spain; 2002. 
[15] Hepworth P, Trow J, Hill V. User controlled settings in noise mapping software - The effects on calculation speed and accuracy. Euronoise Tampere, Finland; 2006.

[16] van Vanda $\mathrm{EH}$, Stapelfeldt $\mathrm{H}$. Implementing prediction standards in calculation software - the various sources of uncertainty. Forum Acusticum. Budapest, Hungary; 2005.

[17] Li J, Heap AD. A review of spatial interpolation methods for environmental scientists, Geoscience Australia, Record 2008/23 ed., 1st, Canberra, Australia; 2008.

[18] ESRI Inc., Arcgis 9.2 Desktop Help, 2009; 2007.

[19] European Commission Working Group - assessment of exposure to noise, Good practice guide for strategic noise mapping and the production of associated data on noise exposure. Version 2, WG-AEN 004.2007; 2007.

[20] Boeker ER, Dinges E, He B, Felming G, Roof C], Gerbi PJ, et al. Integrated noise model (INM) version 7.0 technical manual, FAA-AEE-08-01; 2008

[21] Lewis D, Catlett J. Heterogeneous uncertainty sampling for supervised learning. In: Machine learning: proceedings of the eleventh international conference; 1994. p. $148-56$
[22] Cohn D, Atlas L, Ladner R. Improving generalization with active learning. Mach Learn 1994;15:201-21.

[23] International organization for standardization. Working group 1 of the joint committee for guides in metrology, Evaluation of measurement data - Guide to the expression of uncertainty in measurement, ISO. Geneva, Switzerland; 2008.

[24] Deutsches Institut für Normung (DIN) NA 001 BR-02 SO Comittee, DIN 45687 Acoustics - software products for the calculation of the sound propagation outdoors - quality requirements and test conditions; 2006.

[25] Asensio C, Ruiz M, Pavón I, Ausejo M, Recuero M. Uncertainty in noise maps isolines: the effect of the sampling grid. Acta Acustica United with Acustica; Accepted for Publication..

[26] Paviotti M. Necessary quality of the input data in the noise mapping methods as a function of expected output results. Noise mapping according to the 2002/ 49/EC. Target quality and input requirements. Ispra, Italy; 2009.

[27] Stapelfeldt Ingenieurgesellschaft mbH, Lima 5.2 technical manual; 2008. 